\title{
Impact of FBAR design on its sensitivity as in-liquid gravimetric sensor
}

\author{
T. Mirea , J. Olivares', M. Clement , E. Iborra
}

Keywords:

AlN-based thin film acoustic resonator

Liquid

Density-viscosity

Sensitivity

\begin{abstract}
A B S T R A C T
In contrast to quartz crystal microbalances, the sensitivity of thin film bulk acoustic wave resonators (FBARs) is strongly dependent on all films comprising the multilayered structure. Previous studies proved that placing low acoustic impedance materials at the sensing surface of longitudinal-mode FBARs operating in air can enhance their mass sensitivity by modifying the energy trapped at the sensing surface. Here we investigate if the in-liquid sensitivity to density-viscosity and mass of shear-mode AlN-based solidly mounted resonators displays a similar dependence on the device configuration. By using the finite element simulation method accompanied by experimental verifications we demonstrate that for a given value of the resonant frequency, the sensitivity can be boosted by a proper design of the devices. The results can be of application to in-liquid physical sensors or to gravimetric chemical or biological sensors.
\end{abstract}

\section{Introduction}

Modern activities in many economic sectors would be unconceivable without sensors. Their market covers a wide range of applications, from engine monitoring in the aerospace sector to diagnosis in the healthcare area. One of the most demanded types of sensors are those able to operate in liquid media, either monitoring the physical properties of some liquids $[1,2]$ or acting as chemical sensors or biosensors $[3,4]$ that respond to biochemical reactions in liquid solutions (i.e. corporal fluids or buffer solutions). Biosensors have attracted most of the attention of the scientific community owing to their prospects as point-of-care diagnostics aid in the healthcare area. Much effort is being devoted to the development of bio-chip (or lab on a chip) platforms [5-7], which makes their use easy and affordable by non-expert users.

Among the various technologies that can be used to manufacture devices for sensing applications, gravimetric sensors based on thin film electroacoustic resonators have emerged as a promising choice [8-10]. Compared with other acoustic devices, such as quartz crystal microbalances (QCM) or surface acoustic wave (SAW) devices, thin film resonators not only display smaller sizes and lower power consumption but also offer the possibility of being configured as arrays or monolithically integrated with RF ICs, thus providing more complex functionalities. The most studied devices within this family are the thin film bulk acoustic wave resonator (FBAR) [11-15] and the Lamb wave resonator (LWR) [16-18], both of them employing either $\mathrm{ZnO}$ or AlN piezoelectric films displaying wurtzite crystal structures with a strong $c$-axis preferred orientation. Particularly, in FBARs acoustic waves propagate through the bulk of the piezoelectric film sandwiched between two electrodes; the acoustic wave is confined in the piezoelectric slab either by using suspended structures (suspended-FBAR) or by means of acoustic reflectors [19], which prevents the acoustic energy from leaking to the substrate. The latter is referred to as solidly mounted resonator (SMR). Depending on the crystal orientation of the wurtzite microcrystals in the films one can excite longitudinal thickness modes (if the $c$-axis is parallel to the electric field), shear thickness modes (if the $c$-axis is perfectly normal to the electric field), or both (if the $c$-axis has a component parallel to the field and a component normal to the filed). Longitudinal modes consist of compressional waves in which the particle motion follows the direction of the wave propagation; these modes are especially suited for gas sensing applications. On the contrary, shear modes are waves where the particle motion is transversal to the direction of wave propagation, which is particularly appropriate for sensing application in liquid environments, since shear waves do not couple to this media [20,21].

The sensitivity to mass attachment or liquid properties (density and viscosity) of QCMs can be derived from Sauerbrey's [22] or Kanazawa and Gordon's [23] equations, being in both cases directly related to resonant frequency and only influenced by the quartz plate properties, as the effect of the thin electrodes is negligible. The significant reduction of the thickness of the piezoelectric 
(a)

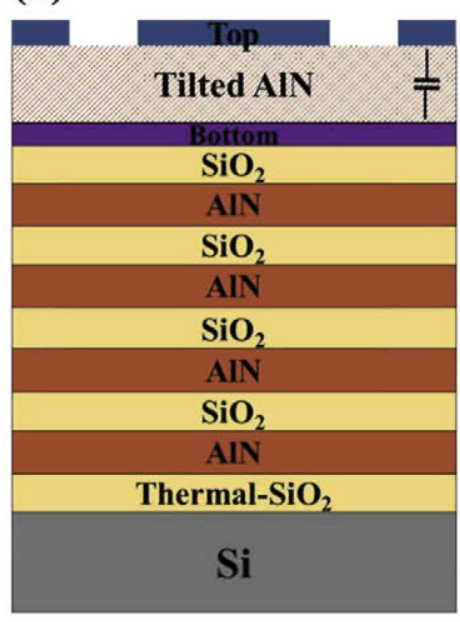

(b)

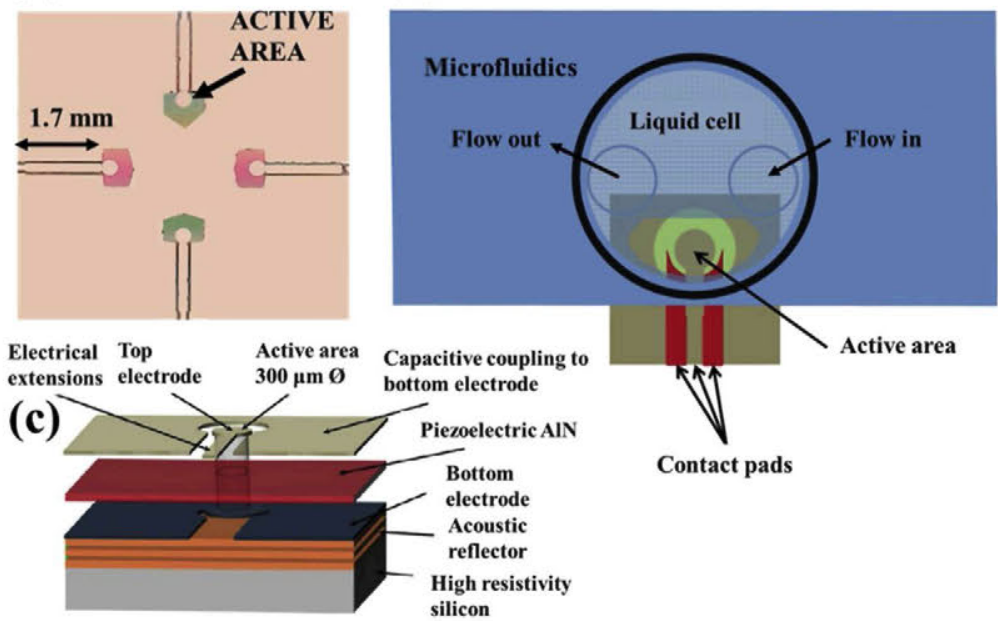

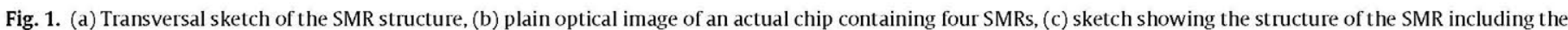
etched bottom electrode below the electrical extensions, and (d) schematic of the microfluidic cell mounted on the active area of the device.

film in FBARs pushes the resonant frequencies towards the $\mathrm{GHz}$ range, which provides with a tremendous increase in sensitivity compared to their QCM predecessors [24,25]. However, the resonant frequency cannot be indefinitely increased to enhance the mass sensor sensitivity, since the readout circuits impose frequency restrictions. In FBARs the sensitivity cannot be derived from the former equations anymore; instead, we need to consider the multilayered structure where the properties of each layer may have a significant influence [26,27]. Particularly, the distribution of the acoustic energy within the structure strongly affects the sensitivity at the active surface. For example, previous investigations [26] prove that the addition of a layer of low acoustic impedance (e.g. $\mathrm{SiO}_{2}$ ) on the sensing surface of suspended-FBARs operating in air produces an increase of their mass sensitivity. The authors carry out an in-dep analysis of the of the mass sensitivity of the fundamental longitudinal mode and its first harmonic and attribute the enhancement of the pure mass sensitivity to an energy trapping effect in the near surface region. They also theoretically asses the effect of the added layer on the energy dissipation to the liquid, although without experimental verification. A similar effect has been reported in FBARs operating in air by using low acoustic impedance electrodes of appropriate thicknesses [27].

In this work we investigate to what extent the sensitivity of shear-mode SMRs operating in liquid environments at the maximum frequency imposed by the external readout circuit can be further enhanced by modifying the design of the device (i.e. electrodes materials and thicknesses). We assess the in-liquid sensitivity of shear-mode SMRs with different structures operating as density and viscosity sensors or as gravimetric biosensors. The experimental results are compared with finite element method (FEM) simulations that predict the behavior of the devices. Differences in frequency shift variations of almost $1000 \mathrm{ppm}$ due to large variations in density and viscosity are observed in devices tuned to similar resonant frequencies but different multilayered structures.

\section{Methods}

\subsection{Device fabrication and measurement set-up}

The structure of our SMRs correspond to Fig. 1a. Metal/tiltedAlN/Metal sandwiches of different thickness and materials were sputtered on top of fully insulating acoustic reflectors made of seven alternating layers of $\mathrm{SiO}_{2} / \mathrm{AlN}$ sputtered over highly resistive silicon wafers. The tilted AIN film was sputtered on top of the bottom electrode through a two stage process involving the initial growth of a thin AIN seed layer $[28,29]$ followed by an off-axis deposition of the active layer. The acoustic reflector was designed to properly confine (reflect) the acoustic energy of the shear mode operating at around $2 \mathrm{GHz}$. This reflector was kept the same for all the devices. Since our purpose was to study the influence of the nature and thickness of the materials composing the piezoelectric stack on the device sensitivity, we varied the relative thickness of the metallic electrodes and AlN active layers (as well as the nature of the electrodes) while adjusting the resonant frequency $f_{\mathrm{r}}$ to an almost constant value close to $2 \mathrm{GHz}$. We studied the five structures described in Table 1.

To allow testing the response of the devices through a microfluidic system, the electrical contacts to the top electrode were extended $1.7 \mathrm{~mm}$ far away from the resonator active area to ease contacting the devices outside the fluidic system with the RF probes (Fig. 1b-d). The bottom electrode located below the extensions of the top electrode was removed to minimize the presence of parasitic capacitances and inductances, hence the use of fully-insulating reflectors together with non-conducting substrates, which avoids capacitive coupling between the top electrode and other metallic underlying layers [30].

We measured the SMRs sensitivities to the physical properties of the liquid by exposing the sensors to ethylene glycol-water solutions of variable density $(\rho)$ and viscosity $(\eta)$. Ethylene glycol weight in water was varied from $0 \%$ to $72 \%$, which led to $\rho$ values in the range of $995 \mathrm{~kg} / \mathrm{m}^{3}-1087 \mathrm{~kg} / \mathrm{m}^{3}$ and $\eta$ values in the range of $0.972 \mathrm{mPa} \cdot \mathrm{s}-4.76 \mathrm{mPa} \cdot \mathrm{s}$ [31]. To study the in-liquid gravimetric sensitivity of the sensors we experimentally detected the binding of bovine serum albumin (BSA) diluted to $0.1 \%$ in a phosphate buffered saline (PBS) solution.

The devices were electrically evaluated by measuring the $S_{11}$ scattering parameter with a network analyzer during their in-liquid operation. The fluidic system consisted in a poly-dimethylsiloxane (PDMS) chamber where the liquids were recirculated with a peristaltic pump (Fig. 1d). As mentioned, the SMRs were electrically contacted through their $1.7 \mathrm{~mm}$ extensions with a $150 \mu \mathrm{m}$-pitch RF-probe connected to the network analyzer. The device response (variations of its resonant frequency) was monitored by tracking the maximum of the real part of the admittance $(\operatorname{Re}(\mathrm{Y}))$ curve through a specifically designed LabView ${ }^{\circledR}$ application. The proce- 
Table 1

Description of the studied SMR structures.

\begin{tabular}{|c|c|c|c|c|}
\hline Structure & Bottom electrode material/ thickness [nm] & AIN thickness [nm] & Top electrode material/ thickness [nm] & Resonant frequency [GHz] \\
\hline $\mathrm{a}$ & Mo / 100 & 900 & Mo / 150 & 2.007 \\
\hline b & Mo / 150 & 900 & Mo/ 100 & 2.010 \\
\hline c & Mo/ 100 & 1000 & $\mathrm{Mo} / 100$ & 2.025 \\
\hline d & $\mathrm{Mo} / 100$ & 900 & $\mathrm{Mo} / 100$ & 2.136 \\
\hline $\mathrm{e}$ & Mo / 100 & 1000 & $\mathrm{Al} / 100$ & 2.261 \\
\hline
\end{tabular}

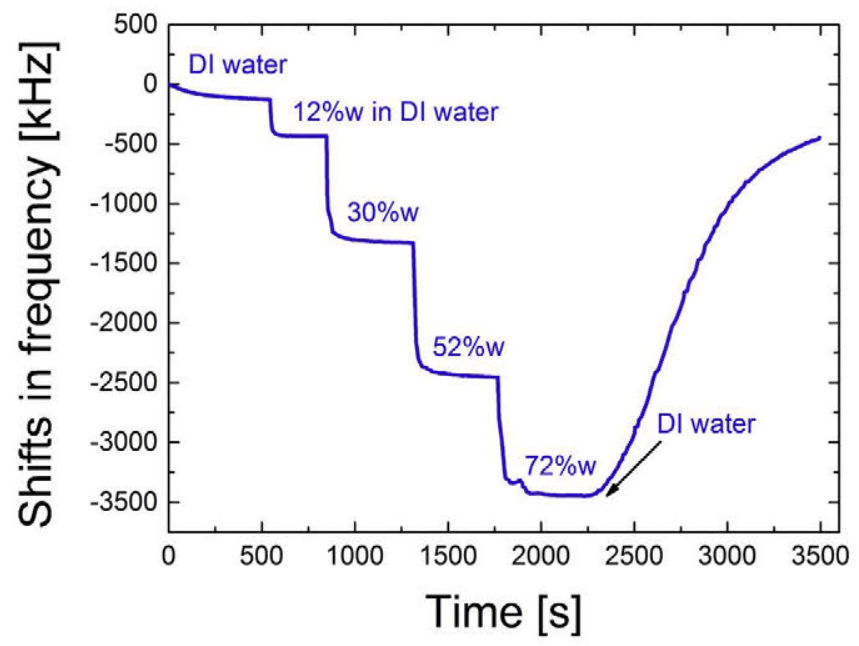

Fig. 2. Typical response of a device with increasing concentration of ethylene glycol $\%$ in pure water (\%w stands for weight percent of solute in water).

dure was based on acquiring $\operatorname{Re}(\mathrm{Y})$ around the resonant frequency with a bandwidth of $20 \mathrm{MHz}(\tilde{4} \mathrm{kHz}$ frequency spacing using 5000 data points) and fitting it to a rational function to accurately measure the frequency at the maximum, which was achieved by deriving the function and calculating the zero cross. This procedure allowed tracking the resonant frequency with a noise below $1 \mathrm{kHz}$ peak to peak, however, it does not evaluate the instabilities due to other external factors such as temperature. Fig. 2 shows the typical response curve of a detection process during which the concentration of an ethylene glycol solution diluted in water was sequentially varied, which produced negative frequency shifts upon introduction of the different solutions. As observed, the solutions were sequentially fed to the chamber, starting with the less viscous and dense one. At the end of the exposure, water was thoroughly flown to clean the device, which was confirmed by the final recovery of its initial resonant frequency.

\subsection{FEM simulations}

To predict the experimental results, we carried out a 2D finite element analysis to theoretically assess the behavior of our devices towards $\rho$ and $\eta$ variations in the liquid. We used a previouslydesigned FEM model [32-34] designed to accurately account for the acoustic coupling to the liquid through shear stresses. The structures we employed for the simulations were single cells with lateral periodic conditions (Fig. 3). In other words, we applied periodic continuity on the lateral interfaces since we were not interested in the possible spurious modes propagating parallel to the surface of the structures but rather in the physical interaction of the shear mode with the liquid interface in the perpendicular direction. The cells included perfectly matched layers (PML) to simulate the total absorption of the outgoing waves in the liquid domain. For the shear mode operation, the AlN domain was defined using a system rotated $24^{\circ}$ about an out-of-plane axis, in agreement with the actual $c$-axis tilt angle of the AlN films. Thicknesses of all materi-

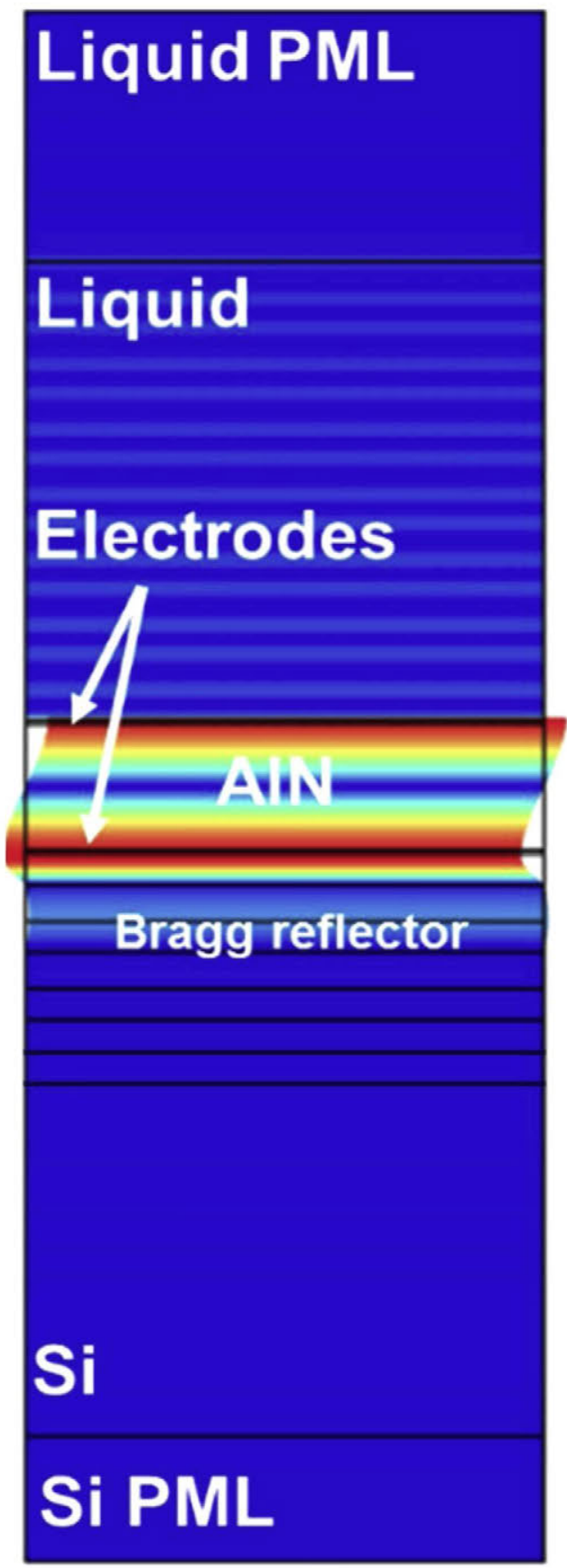

Fig. 3. Displacements with deformation of an SMR in contact with liquid obtained by FEM.

als composing the devices were set according to Table 1 . With this model we can extract the frequency shifts towards different $\rho$ and $\eta$ variations and mass deposition, as well as estimate the losses induced in the devices. The resonant frequency was calculated as the frequency at the maximum of the $\operatorname{Re}(\mathrm{Y})$ curve, while the losses 


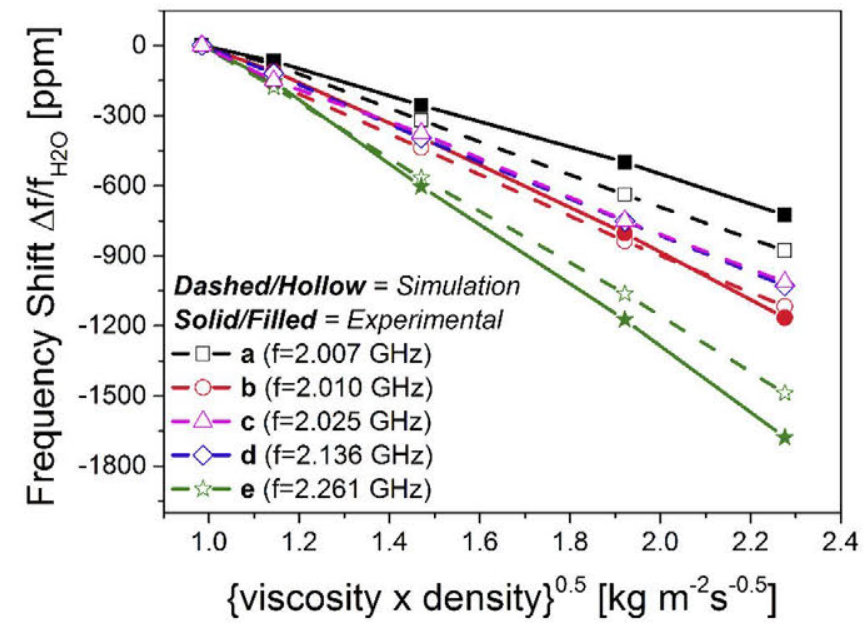

Fig. 4. Simulated and experimental normalized shifts in frequency of the five different structures, described in Table 1 , towards increasing $\rho$ and $\eta$ products.

Table 2

Measured and simulated sensitivities to $(\rho \eta)^{0.5}$.

\begin{tabular}{lll}
\hline Structure & Measured $\left[\mathrm{ppm} /\left(\mathrm{kg} \cdot \mathrm{m}^{-2} \cdot \mathrm{s}^{-0.5}\right)\right]$ & Simulated $\left[\mathrm{ppm} /\left(\mathrm{kg} \cdot \mathrm{m}^{-2} \cdot \mathrm{s}^{-0.5}\right)\right]$ \\
\hline a & 570 & 580 \\
$\mathrm{~b}$ & 919 & 877 \\
$\mathrm{C}$ & $\mathrm{n} / \mathrm{a}$ & 797 \\
$\mathrm{~d}$ & $\mathrm{n} / \mathrm{a}$ & 797 \\
$\mathrm{e}$ & 1320 & 1170 \\
\hline
\end{tabular}

estimated from the $\operatorname{Re}(\mathrm{Y})$ amplitude value. This last has the same sensitivity as the quality factor of the device.

\section{Results and discussion}

We started by simulating the responses of all the SMR structures towards variations of $\rho$ and $\eta$ in the liquids. Following the conventional plot described in [32], their simulated frequency shifts are depicted in Fig. 4 as a function of $(\rho \eta)^{0.5}$, along with the experimental results corresponding to the structures displaying most different sensitivities ( $a, b$ and e). The simulated and measured sensitivities to $(\rho \eta)^{0.5}$ are summarized in Table 2. Firstly, we note a reasonable agreement between simulations and experiments. Secondly, we observe an enhanced sensitivity to $\rho$ and $\eta$ if we use $\mathrm{Al}$ instead of Mo as top electrode, having the former one a lower acoustic impedance than Mo, and when the Mo top electrode thickness is reduced from $150 \mathrm{~nm}$ to $100 \mathrm{~nm}$. The reason for this is a redistribution of the energy within the acoustic cavity, which leads to an increased displacement field (or energy concentration) at the top electrode surface in contact with the liquid. Note that this is not a second order effect, as the values of the sensitivity to $\rho$ and $\eta$ product are more than doubled from one case to the other.

This is in agreement with previously reported results where only pure mass sensitivity in air was considered [26]. Indeed, if one considers the transmission coefficient of the wave amplitude at the AlN/top electrode interface, which is defined as:

$T=\frac{\left(2 \cdot Z_{\text {AIN }}\right)}{\left(Z_{\text {AIN }}+Z_{\text {top }}\right)}$

being $Z_{\text {AIN }}$ the specific acoustic impedance of AlN and $Z_{\text {top }}$ that of the top electrode, it is clear from Table 3 (acoustic impedance values) that the amplitude of the transmitted wave from AlN to the top electrode is increased when using $\mathrm{Al}(T=1.42)$, and decreased when using Mo $(T=0.72)$. These values explain why we have a greater amount of displacement involved in the sensing mechanism when using $\mathrm{Al}$ as top electrode and thus a sensitivity enhancement.
Table 3

Materials properties. Acoustic impedance calculated from the product of density and shear acoustic velocity. Acoustic velocity was calculated following a specific method [35].

\begin{tabular}{llll}
\hline Material & Shear acoustic velocity $[\mathrm{m} / \mathrm{s}]$ & Density $\left[\mathrm{kg} / \mathrm{m}^{3}\right]$ & Z [Rayl] \\
\hline AIN & 6170 & 3300 & $2.03 \cdot 10^{7}$ \\
Al & 3040 & 2700 & $8.21 \cdot 10^{6}$ \\
Mo & 3450 & 10300 & $3.55 \cdot 10^{7}$ \\
\hline
\end{tabular}

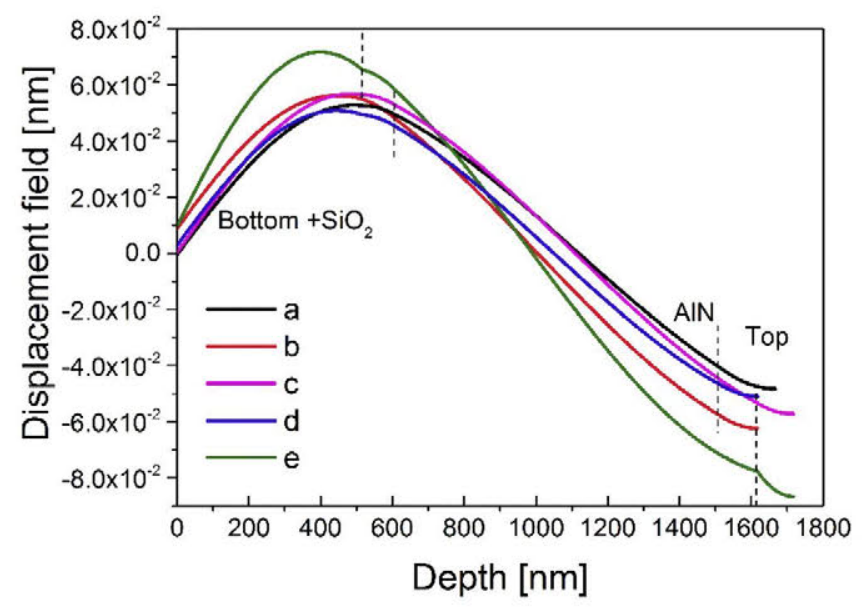

Fig. 5. Simulated displacement fields at resonance in air for all structures from Table 1.

It is interesting to note that structures (b), (c) and (d), where the thickness of the top electrode is kept constant to $100 \mathrm{~nm}$, do not show any significant sensitivity variation despite the fact that both the thickness of the AlN active layer and the bottom electrode are varied. This is very likely due to the fact that the energy concentration at the sensing interface barely varies and is little affected by the structural changes of the piezoelectric stack.

In spite of the fact that we intended to keep the resonant frequency $f_{\mathrm{r}}$ constant to around $2 \mathrm{GHz}$, some structures displayed a deviation from this value of around $10 \%$. In order to check whether the variations in $f_{\mathrm{r}}$ between structures (a) and (e) might account for the observed sensitivity changes, as would be the case of QCMs, we performed a simplistic calculation to assess to what extent the frequency shift could be attributed to the value of $f_{\mathrm{r}}$. The frequency shift was calculated according to Kanazawa and Gordon's [23] equation defined as:

$\Delta f=-f_{0} \frac{3}{2}\left(\frac{\rho_{l} \eta_{l}}{\pi \rho_{q} \mu_{q}}\right)^{\frac{1}{2}}$

where $\Delta f$ is the frequency shift, $f_{0}$ the initial resonant frequency, $\rho_{l}$ and $\eta_{l}$ the density and viscosity of the liquid and $\rho_{q}$ and $\mu_{q}$ the density and shear elastic modulus of the piezoelectric material, respectively (here $q$ stands for quartz taken from the [23]). We could suppose that $\rho_{l} \eta_{l} / \pi \rho_{q} \mu_{q}$ is constant and consider the case corresponding to the maximum value of $(\rho \eta)^{0.5}$ of the experimental solution (2.3). In this case, the measured frequency shifts are around $750 \mathrm{ppm}$ for structure (a) (i.e. $1.505 \mathrm{MHz}$ ) and $1650 \mathrm{ppm}$ for structure (e) (i.e. $3.73 \mathrm{MHz}$ ), which corresponds to an increment by a factor of 2.47 . The calculation of the increment in the frequency shift with eq. (2) yields a value of only 1.19, significantly lower than the measured one, suggesting the increased sensitivity is mainly due to an energy redistribution in the multilayered structure. In order to verify this effect we extracted from the FEM results the displacements at resonance for each design. The displacement fields in air corresponding to the shear (or parallel to the surface) component are plotted in Fig. 5. Indeed, these corroborate the sen- 


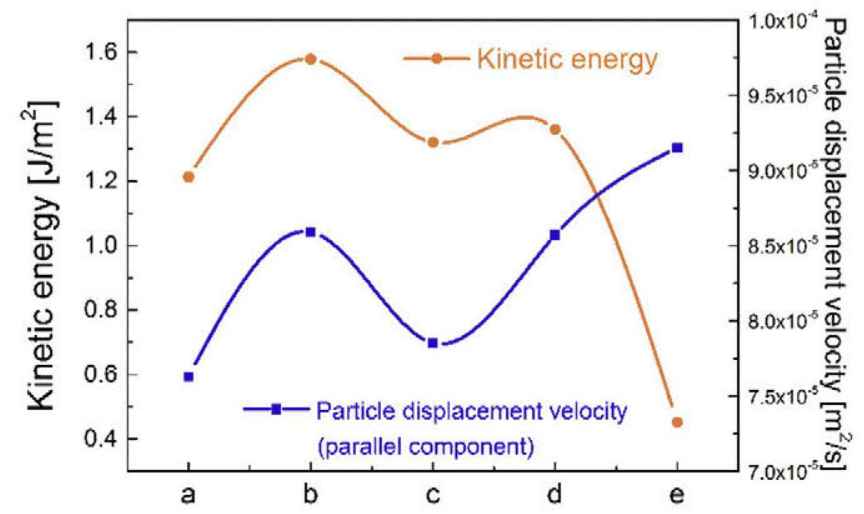

Fig. 6. Simulated kinetic energy densities at the top surface of all structures in air and the corresponding particle displacement velocities of the parallel component at the same surface.

sitivity results from Fig, 4, yielding the most sensitive structure (i.e. structure e) the larger displacement at the top surface, which contacts the liquid.

Additionally, to explain the increased sensitivity effect we also extracted the kinetic energy density at the top surface of each design. Particularly, the calculation consisted on its integration along the top surface line, since we simulated a $2 \mathrm{D}$ model. The corresponding values for each structure are plotted in Fig. 6. As we observe, while for the structures with Mo top electrodes we get higher kinetic energy for the more sensitive devices, structure (e), with the Al top electrode, has the lowest kinetic energy. To explain this, the parallel component of the particle velocity at the top surface shows a better insight of the phenomena. The particle displacement velocities for each design are also plotted in Fig. 6, where we see that the higher the displacement velocity at the surface, the more sensitive the structure is. The reason why structure (e) has lower kinetic energy but higher displacement velocity at the surface is based on the fact that Al has lower mass density and lower shear acoustic velocity. By considering Eq. (3):

$E_{k}=\frac{\left(\rho v^{2}\right)}{2}=\frac{\left(Z_{a c} v^{2}\right)}{\left(2 v_{a c}\right)}$

where $\rho$ is the density of the material, $v$ the displacement velocity, $Z_{a c}$ the acoustic impedance of the material, and $v_{a c}$ the velocity of the acoustic wave propagation in the material, results from Fig. 6 can be easily derived.

Higher particle displacement velocity at the top surface implies that when such surface is contacted by the liquid the acoustically coupled thin layer of liquid (where the evanescent acoustic wave in the liquid is concentrated, i.e. the penetration depth) acquires at the interface with the resonator the same displacement velocity, with the subsequent damping of the shear wave. The kinetic energy of the acoustically coupled thin liquid layer will be higher if the velocity transmitted from the resonator is higher, hence more energy lost and larger shift in frequency to compensate it since the potential energy of the resonator does not vary and kinetic and potential energies equal at resonance [8].

The same effect was studied for different thicknesses of a Mo top electrode, while maintaining the same thickness of AlN and same bottom electrode. Particle displacement velocities are plotted in Fig. 7, where it can be observed that the thicker the Mo top electrode, the lower the displacement velocity at the surface, therefore less sensitive structures.

The sensitivity enhancement achieved by modifying the design of the layered structure is unavoidably accompanied by an increase of the losses to the liquid, as clearly shown in Fig. 8, which is justified by the larger energy coupling taking place between the most

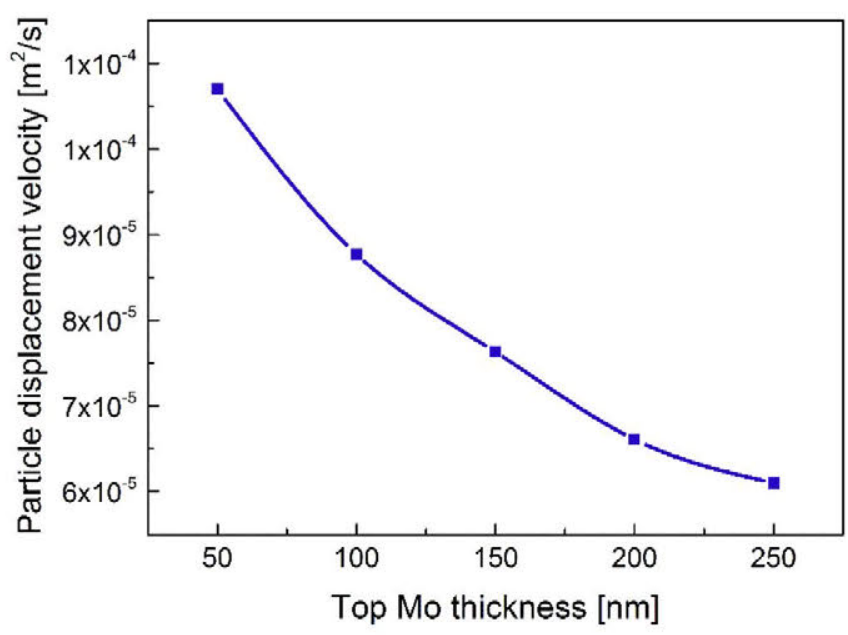

Fig. 7. Simulated particle displacement velocities at the top surface of structures with different Mo top electrode thicknesses.

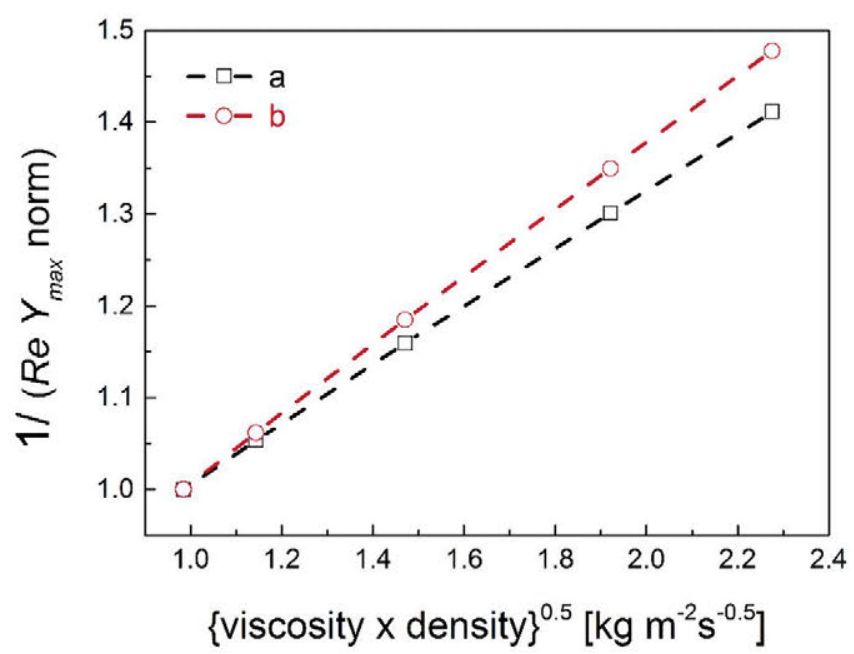

Fig. 8. FEM simulation of the losses (inverse of the admittance peak normalized to its value in pure water) for structures (a) and (b). The losses raise with $\rho$ and $\eta$.

Table 4

FEM results on the mass sensitivity of the devices while immersed in water upon deposition of a $50 \mathrm{~nm}$-thick layer of $\mathrm{SiO}_{2}$.

\begin{tabular}{ll}
\hline Structure & Simulated $\left[\mathrm{Hz} \mathrm{cm}^{2} / \mathrm{ng}\right]$ \\
\hline a & 1956 \\
b & 2459 \\
c & 2328 \\
d & 2380 \\
e & 4483 \\
\hline
\end{tabular}

sensitive devices and the liquid. This produces a decrease in the quality factors of the devices.

As a final experiment, we tested the in-liquid mass sensing ability of our devices. Firstly, we present simulated results regarding the pure mass sensitivity of the devices while immersed in water towards the addition of a $50 \mathrm{~nm}$-thick $\mathrm{SiO}_{2}$ film (i.e. $11 \mu \mathrm{g} / \mathrm{cm}^{2}$ ) on their top surface (see Table 4). These FEM results were previously validated with a test SMR and the deposition of a $50 \mathrm{~nm}$-thick $\mathrm{SiO}_{2}$ in air, for both experiment and simulation, obtaining a deviation of $3 \%$.

As a realistic experiment, we assessed the sensors performance upon exposing their active area to a solution of bovine serum albumin (BSA). This protein is known for its high chemical affinity with different surfaces, being widely used as blocking agent in 


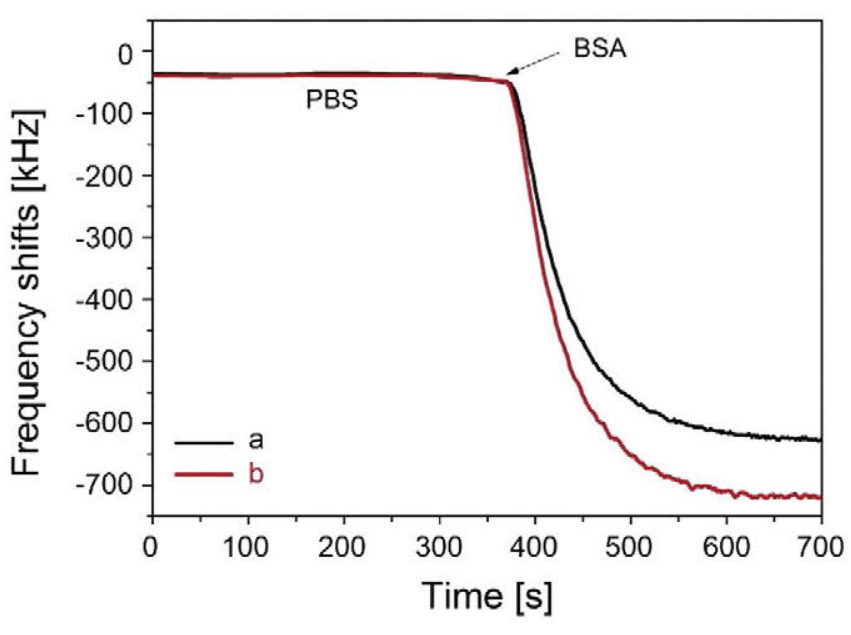

Fig. 9. Shifts in frequency for structures (a) and (b) upon attachment of BSA.

bio-functionalization protocols. With this purpose, we used a $0.1 \%$ concentration of BSA in PBS. In Fig. 9 we plot the frequency shifts upon attachment of the BSA to the surface of structures (a) and (b) terminated with $150 \mathrm{~nm}$-thick and $100 \mathrm{~nm}$-thick top electrodes, respectively. As expected, the experiment reveals that the reduction of the Mo electrode thickness from $150 \mathrm{~nm}$ to $100 \mathrm{~nm}$ gives rise to a more sensitive device, despite the fact that the whole structure has been modified to keep the resonant frequency almost constant. However, by extracting the attached mass of BSA using the calculated mass sensitivities from Table 4 we get $294 \mathrm{ng} / \mathrm{cm}^{2}$ for device (a), and $274 \mathrm{ng} / \mathrm{cm}^{2}$ for device (b), a difference lower than expected. We attribute this discrepancy to possible non-uniform attachments of BSA.

\section{Conclusions}

The main conclusions of the work are the following:

- The structure (materials and thicknesses) of the near active region of SMRs significantly affects their in-liquid sensitivity, owing to the energy redistribution inside the resonant cavity, particularly at the sensing interface. Slight changes in the structure of SMRs may increase their sensitivity to a much greater extent than increasing the resonant frequency (i.e. reducing the thickness of the active piezoelectric). Actually, as the electronic read-out circuits conditioning the electric signal of the resonators may impose limits on the operation frequency of the sensors, a good design of their structure provides sensitivity enhancement without unnecessarily increasing the resonant frequency and therefore the noise.

- As in suspended-FBARs operating in longitudinal modes for gravimetric gas detection, reducing the acoustic impedance of the top electrode of shear-mode SMRs (present at the sensing interface) increases their sensitivity to density and viscosity and to mass in liquid. Similarly, the same enhancement is produced when reducing the thickness of high impedance electrodes.

- The in-liquid sensitivity enhancement is accompanied by an increase of the losses towards the surrounding fluid, hence a trade-off has to be reached between the sensor sensitivity and the ability of our read-out electronic circuit to handle the signal. Nevertheless, we have demonstrated that the sensitivity increase dominates.

\section{Acknowledgments}

This work was partially supported by Ministerio de Economía y Competitividad del Gobierno de España through project MAT201345957-R.

\section{References}

[1] B. Jakoby, M.J. Vellekoop, Physical sensors for liquid properties, IEEE Sens. J. 11 (2011) 3076-3085, http://dx.doi.org/10.1109/JSEN.2011.2167716.

[2] T. Manzaneque, V. Ruiz-Díez, J. Hernando-García, E. Wistrela, M. Kucera, U. Schmid, J.L. Sánchez-Rojas, Piezoelectric MEMS resonator-based oscillator for density and viscosity sensing, Sens. Actuators A Phys. 220 (2014) 305-315, http://dx.doi.org/10.1016/j.sna.2014.10.002.

[3] J.P. Smith, Medical and biological sensors: a technical and commercial review, Sens. Rev. 25 (2005) 241-245, http://dx.doi.org/10.1108/ 02602280510620060 .

[4] B.R. Eggins, Chemical Sensors and Biosensors, J. Wiley, 2002.

[5] F. Liu, a N. Nordin, F. Li, I. Voiculescu, A lab-on-chip cell-based biosensor for label-free sensing of water toxicants, Lab Chip 14 (2014) 1270-1280, http:// dx.doi.org/10.1039/c3lc51085a.

[6] Z. Wang, H. Wu, D. Fine, J. Schmulen, Y. Hu, B. Godin, J.X.J. Zhang, X. Liu, Ciliated micropillars for the microfluidic-based isolation of nanoscale lipid vesicles, Lab Chip 13 (2013) 2879-2882, http://dx,doi,org/10,1039/ c3lc41343h.

[7] K. Mitsakakis, A. Tserepi, E. Gizeli, Integration of microfluidics with a love wave sensor for the fabrication of a multisample analytical microdevice, J. Microelectromech. Syst. 17 (2008) 1010-1019, http://dx.doi.org/10.1109/ JMEMS.2008.927173.

[8] D.S. Ballantine, S.J. Martin, A.J. Ricco, G.C. Frye, H. Wohltjen, R.M. White, E.T. Zellers, Acoustic Wave Sensors, Elsevier, 1997, http://dx.doi.org/10.1016/ B978-0-12-077460-9.50012-5.

[9] I. Voiculescu, A.N. Nordin, Acoustic wave based MEMS devices for biosensing applications, Biosens. Bioelectron. 33 (2012) 1-9, http://dx.doi.org/10.1016/j. bios.2011.12.041.

[10] I. Katardjiev, V. Yantchev, Recent developments in thin film electro-acoustic technology for biosensor applications, Vacuum. 86 (2012) 520-531, http://dx. doi.org/10.1016/J.VACUUM.2011.10,012.

[11] K.M. Lakin, Thin film resonator technology, IEEE Trans. Ultrason. Ferroelectr. Freq. Control 52 (2005) 707-716, http://dx.doi.org/10.1109/TUFFC.2005. 1503959.

[12] J. Weber, W.M. Albers, J. Tuppurainen, M. Link, R. Gabl, W. Wersing, M. Schreiter, Shear Mode FBARs as Highly Sensitive Liquid Biosensors, 2006 , http://dx.doi.org/10.1016/j.sna,2006.01.005.

[13] G. Wingqvist, J. Bjurstrom, A. Hellgren, I. Katardjiev, Immunosensor utilizing a shear mode thin film bulk acoustic sensor, Sens. Actuators B Chem. 127 (2007) 248-252, http://dx.doi.org/10.1016/j.snb.2007.07.051.

[14] W. Xu, X. Zhang, S. Choi, J. Chae, A high-quality-factor film bulk acoustic resonator in liquid for biosensing applications, J. Microelectromech. Syst. 20 (2011) 213-220, http://dx.doi.org/10.1109/IMEMS.2010.2093568.

[15] R. Gabl, E. Green, M. Schreiter, H.D. Feucht, H. Zeininger, R. Primig, D. Pitzer, G. Eckstein, W. Wersing, Novel integrated FBAR sensors: a universal technology platform for bio- and gas-detection, Proc. IEEE Sensors 2003 (IEEE Cat. No.03CH37498) 2 (2003) 1184-1188, http://dx.doi.org/10.1109/ICSENS.2003. 1279132.

[16] V. Yantchev, I. Katardjiev, Micromachined thin film plate acoustic resonators utilizing the lowest order symmetric lamb wave mode, IEEE Trans, Ultrason. Ferroelectr. Freq. Control 54 (2007) 87-95 (accessed January 10, 2017) http:// www.ncbi.nlm.nih.gov/pubmed/17225803.

[17] L. Arapan, E. Anderas, I. Katardjiev, V. Yantchev, Sensitivity features of thin film plate acoustic wave resonators, IEEE Sens. J. 11 (2011) 3330-3331, http:/ dx.doi.org/10.1109/JSEN.2011.2158094.

[18] P.j. Stephanou, A.P. Pisano, GHZ contour extensional Mode aluminum nitride MEMS resonators, 2006 IEEE Ultrason. Symp. (2006) 2401-2404, http://dx. doi.org/10.1109/ULTSYM.2006.581.

[19] R. Ruby, Review and comparison of bulk acoustic wave FBAR, SMR technology, 2007 IEEE Ultrason. Symp. Proc. (2007) 1029-1040, http://dx.doi. org/10.1109/ULTSYM.2007.262.

[20] S.J. Martin, G.C. Frye, K.O. Wessendorf, Sensing liquid properties with thickness-shear mode resonators, Sens. Actuators A Phys. 44 (1994) 209-218, http://dx.doi.org/10.1016/0924-4247(94)00806-X.

[21] G. Wingqvist, J. Bjurström, L. Liljeholm, V. Yantchev, I. Katardjiev, Shear mode AIN thin film electro-acoustic resonant sensor operation in viscous media, Sens. Actuators B Chem. 123 (2007) 466-473, http://dx.doi.org/10.1016/j.snb. 2006.09.028.

[22] G. Sauerbrey, Verwendung von Schwingquarzen zur Wagungdiinner Schichten und zur Mikrowagung, Zeitschrift Fur Phys. 155 (1959) 206-222, http://dx.doi.org/10.1007/BF01337937.

[23] K.K. Kanazawa, J.G. Gordon, Frequency of a quartz microbalance in contact with liquid, Anal. Chem. 57 (1985) 1770-1771, http://dx.doi.org/10.1021/ ac00285a062. 
[24] J. Weber, W.M. Albers, J. Tuppurainen, M. Link, R. Gabl, W. Wersing, M. Schreiter, Shear mode FBARs as highly sensitive liquid biosensors, Sens. Actuators A Phys. 128 (2006) 84-88, http://dx.doi.org/10.1016/j.sna.2006.01. 005.

[25] S. Rey-Mermet, R. Lanz, P. Muralt, Bulk acoustic wave resonator operating at 8 $\mathrm{GHz}$ for gravimetric sensing of organic films, Sens. Actuators B Chem. 114 (2006) 681-686, http://dx.doi.org/10.1016/J.SNB.2005.04.047.

[26] G. Wingqvist, V. Yantchev, I. Katardjiev, Mass sensitivity of multilayer thin film resonant BAW sensors, Sens. Actuators A Phys. 148 (2008) 88-95, http:// dx.doi.org/10.1016/j.sna.2008.07.023.

[27] H. Liu, S. Ma, H. Wang, Q, Wang, L. Qin, Electrode effects on mass sensitivity of GaN thin film bulk acoustic wave resonator sensors, 2015 Symp. Piezoelectricity, Acoust. Waves, Device Appl. (2015) 179-183, http://dx.doi. org/10.1109/SPAWDA.2015.7364467.

[28] J. Bjurström, G. Wingqvist, I. Katardjiev, Synthesis of textured thin piezoelectric AIN films with a nonzero c-axis mean tilt for the fabrication of shear mode resonators, IEEE Trans. Ultrason. Ferroelectr. Freq. Control 53 (2006) 2095-2100, http://dx.doi.org/10.1109/TUFFC.2006.149.

[29] M. DeMiguel-Ramos, T. Mirea, M. Clement, J. Olivares, J. Sangrador, E. Iborra, Optimized tilted c-axis AIN films for improved operation of shear mode resonators, Thin Solid Films 590 (2015) 219-223, http://dx.doi.org/10.1016/j. tsf.2015.08.010.

[30] M. DeMiguel-Ramos, T. Mirea, J. Olivares, M. Clement, J. Sangrador, E. Iborra, M. Barba, Influence of the electrical extensions in AIN-BAW resonators for in-liquid biosensors, 2014 Eur. Freq. Time Forum, IEEE (2014) 301-304, http://dx.doi.org/10.1109/EFTF.2014.7331492.

[31] D.R. Lide, CRC Handbook of Chemistry and Physics: a Ready-reference Book of Chemical and Physical Data, CRC Press, 2004

[32] T. Mirea, V. Yantchev, Influence of liquid properties on the performance of So-mode Lamb wave sensors: a theoretical analysis, Sens. Actuators B Chem. 208 (2015) 212-219, http://dx.doi.org/10.1016/j.snb.2014.11.026.

[33] T. Mirea, V. Yantchev, J. Olivares, E. Iborra, Influence of liquid properties on the performance of SO-mode Lamb wave sensors II: experimental validation, Sens. Actuators B Chem. 229 (2016) 331-337, http://dx.doi.org/10.1016/j.snb. 2016.01.131.

[34] T. Mirea, E. Iborra, V. Yantchev, So Lamb wave resonators for in-liquid sensing: promising alternative to shear bulk acoustic wave devices, 2016 Eur. Freq. Time Forum, IEEE (2016) 1-4, http://dx.doi.org/10.1109/EFTF.2016.7477807.

[35] M. DeMiguel-Ramos, T. Mirea, J. Olivares, M. Clement, J. Sangrador, E. Iborra, Assessment of the shear acoustic velocities in the different materials composing a high frequency solidly mounted resonator, Ultrasonics 62 (2015) 195-199, http://dx.doi.org/10.1016/J.ULTRAS.2015.05.017. 